Vladimir Kataev, Candidate of Technical Sciences, Associate Professor, Senior Lecturer, Volgodonsk Engineering and Technical Institute of NRNU MEPhI. E-mail: kataev.v.f @ gmail.com

Igor Sysoyev, Doctor of Technical Sciences, Professor, Director of the Scientific and Educational Center IEEiN of the North Caucasus Federal University. E-mail: eianpisia@yandex.ru

Dmitry Gusev, Candidate of Technical Sciences, Senior Lecturer, Volgodonsk Engineering and Technical Institute of NRNU MEPhI. E-mail: dmon_amail.ru

\author{
Звада Павел Александрович, Пляшко Максим Сергеевич, \\ Миронов Андрей Валерьевич
}

\title{
ОЦЕНКА ВЛИЯНИЙ ПАРАМЕТРОВ ТОКА ВОЗБУЖДЕНИЯ СИНХРОННОГО ГЕНЕРАТОРА НА ПОКАЗАТЕЛИ КАЧЕСТВА ПРОИЗВОДИМОЙ ЭЛЕКТРИЧЕСКОЙ ЭНЕРГИИ
}

В статье рассмотрен вопрос влияний параметров тока возбуждения синхронной машины и вероятных аварийных ситуаций в обмотках возбуждения на показатели качества вырабатываемой электрической энергии. Проведены активные эксперименты по моделированию ситуаций возникновения аварийньх режсимов в обмотках возбуюдения и системах регулирования тока возбуждения синхронного генератора.

Ключевые слова: несинусоидальность; качество электроэнергии; синхронный генератор; токвозбуждения.

\section{Pavel Zvada, Maxim Plyashko, Andrey Mironov ASSESSMENT OF THE IMPACT EXCITATION CURRENT PARAMETERES OF THE SYNCHRONOUS GENERATOR ON THE INDICATORS OF QUALITY OF THE PRODUCED ELECTRIC ENERGY}

The article discusses issues related to the impact of excitation current parameters of a synchronous machine and of possible emergencies in the field windings on the level of the quality of the generated electrical energy. Active experiments on the simulation of emergency conditions in the windings of the excitations and control systems of the excitation current of a synchronous generator are conducted.

Key words: non-sinusoidal, power quality, synchronous generator, excitation current.

Bведениe / Introduction. Целью данной работы является выявление зависимостей качества сигналов напряжения и тока генератора в процессе его эксплуатации при возникновении ситуаций отклонения частоты тока возбуждения в различных диапазонах.

Вопросы качества электрической энергии согласно ГОСТу 32144-2013 [1] актуальны в период развития малой генерации и появления новых типов нагрузки и технических решений в системах управления генераторными установками. К качеству напряжения у потребителя возникает множество требований, наиболее важными из которых является частота напряжения сети, коэффициенты несинусоидальности и доза фликера, т. к. отклонения этих показателей от нормы могут привести к поломке электрооборудования потребителя, а в случае нарушения этих показателей непосредственно на выводах генерирующей установки - к нарушению работы систем регулирования на станции, потере устойчивости генератора и к выходу из синхронизма. 
Обмотки роторов синхронных генераторов получают питание от специальных источников постоянного тока, называемых возбудителями. Современные схемы возбуждения, кроме возбудителя, содержат большое количество вспомогательного оборудования. Совокупность возбудителя, вспомогательные и регулирующие устройства принято называть системой возбуждения. Важнейшими характеристиками систем возбуждения являются: быстродействие - оценивается по выражению 1 , и кратность форсировки - по выражению 2. В обоих случаях определяющим является уровень напряжения.

$$
\begin{gathered}
V=0,632 \cdot \frac{\left(U_{\text {гпот }}-U_{\text {ғном }}\right)}{U_{\text {гном }} \cdot t_{1}} ; \\
k_{\Phi}=\frac{U_{\text {ппот }}}{U_{\text {fном }}} ;
\end{gathered}
$$

Также существенным параметром систем возбуждения оказывается качество выпрямленного тока. Основной характеристикой в рассматриваемом вопросе является пульсация тока или его колебание. Как известно коэффициентом пульсации называют величину, равную отношению амплитудного значения (максимальной величины) переменной компоненты пульсирующей величины к постоянной составляющей. Если представить выпрямленный ток в виде ряда Фурье как сумму постоянной составляющей $X_{0}$ и некоторого числа $(n)$ гармоник, имеющих амплитуды $X_{\min }$, то коэффициент пульсации напряжения $\left(k_{n}\right)$ можно определить по выражению 3 , где $n-$ номер гармоники.

$$
k_{n}=\frac{x_{\min }}{x_{0}} .
$$

Естественно компонента $x_{0}$ считается полезным результатом деятельности выпрямителя, в отличие от пульсаций $x_{\text {min }}$. Если форма пульсаций сложная, то максимальным значением может обладать не первая гармоника, хотя обычно под $k$ понимают ее. Она применяется в расчетах и записывается в технических документах оборудования, выполняющего функции источника питания обмоток возбуждения генераторов и подобных устройств [2]. Для достижения цели исследования необходимо создать ситуации возникновения гармонических составляющих в токе возбуждения синхронной машины и оценить его влияние на показатели качества электрической энергии производимой генератором.

Maтериалы и методы / Materials and methods. Эксперимент по оценке влияния параметров тока возбуждения на показатели качества электроэнергии, производимой генератором. Для проведения эксперимента была собрана схема на основе стенда «Учебная техника», состоящая из модулей «спарка трехфазный генератор и двигатель постоянного тока», «осветительная нагрузка», «источник питания двигателя постоянного тока» и частотомер, подключенный к датчику угловых перемещений. Физическая модель генератора мощностью 50 Вт в трехфазном режиме нагружалась на осветительную нагрузку, соединенную по схеме звезда с нулем, и в результате регулировки тока возбуждения задавался номинальный режим работы генератора по частоте и выдаваемому уровню напряжения. Также был использован универсальный комплект для испытаний реле и пусконаладочных работ - Omicron CMC 356, в качестве источника питания обмотки возбуждения, позволяющего генерировать токи трех фаз с независимо заданными значениями частоты амплитуды и фазы сигнала. Принципиальная схема для проведения данного эксперимента представлена на рис. 1.

Проводились две группы опытов. Первая группа характеризует ситуации возникновения пульсаций в сигнале тока возбуждения с последующим влиянием на работу генератора. Для этого опыта использовались обмотки возбуждения F1-FN и F2-FN, образующие контур F1-F2, соответственно в системе генерации токов возбуждения задавались направлением фазных токов от 
OMICRON CMC 356 [7], обеспечивая протекание результирующего тока по требуемому контуру (рис. 2a). Вторая серия опытов направлена на имитацию ситуации пробоя изоляции обмотки возбуждения и возникновения тока подпитки (инжекции тока) частотой от 10 до 500 Гц (рис. 2б). Измерение токов и напряжений в сериях опытов производились модулями измерения напряжения NI-9225 [8] и тока NI-9227 [9] точностью 24 бита и с частотой дискретизации 25 тысяч измерений в секунду, объединенных в шасси cDaq-9172 с USB интерфейсом.

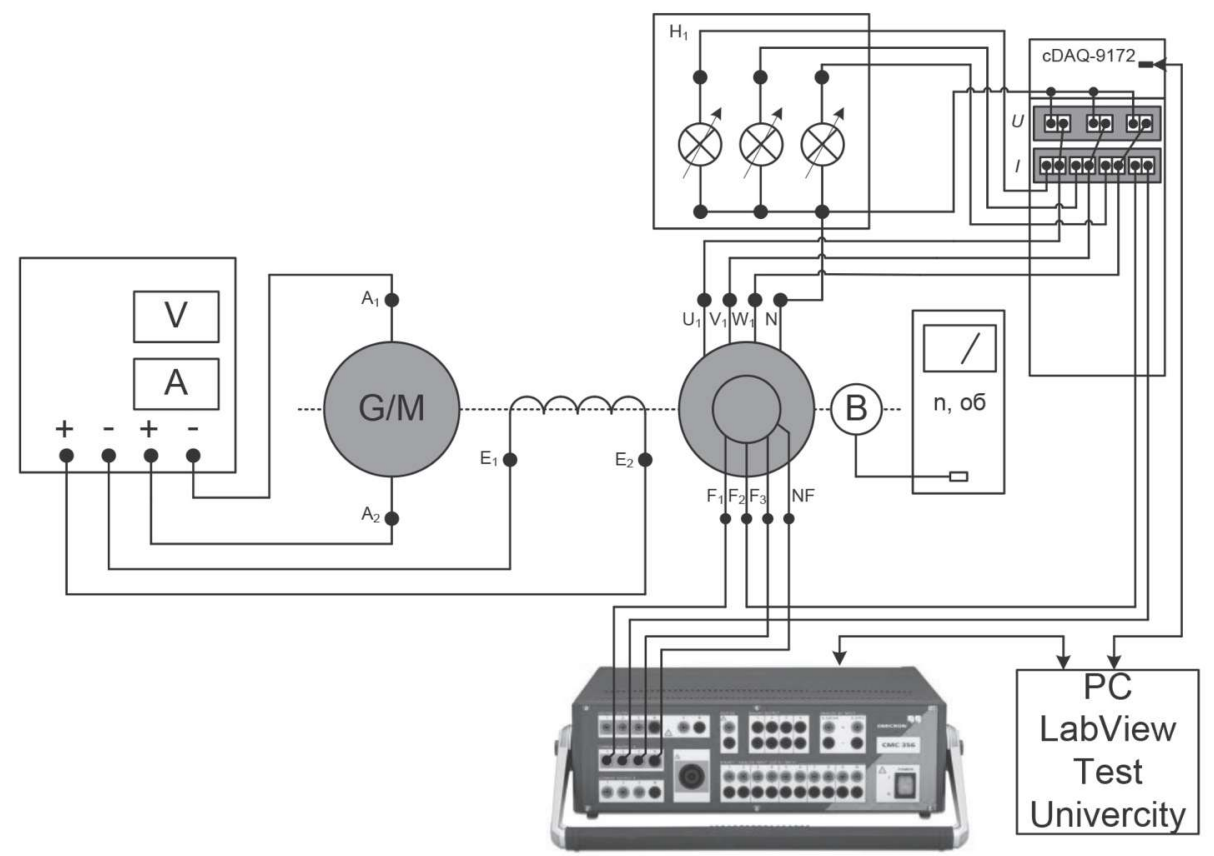

Рис. 1. Принципиальная схема экспериментальной установки

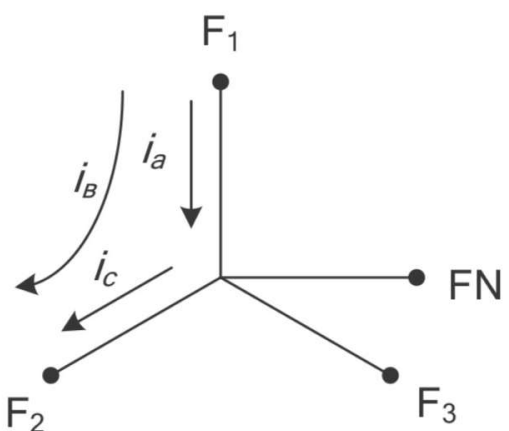

a)

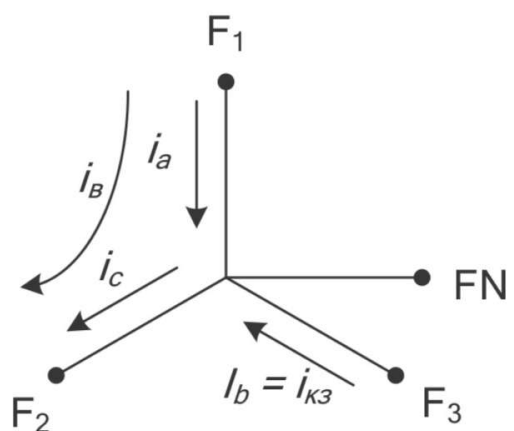

б)

Рис. 2. Схема организации направлений фазных токов:

а) первый эксперимент; б) второй эксперимент

Обработка полученных в результате замеров данных выполняется на предмет получения коэффициентов несинусоидальности сигналов напряжения и тока нагрузки генератора $[10,11]$ при помощи разработанного приложения в графической среде разработки Labview. 
Результаты и обсужсдение / Results and discussion. Для выявления зависимости было проведено два эксперимента, по результатам которых для каждой отдельной величины, а именно тока и напряжения, были рассчитаны коэффициенты несинусоидальности.

Первый эксперимент был проведён для нормального режима работы генератора, с фиксированным значением тока возбуждения, но с частотой, варьируемой от 0,1 до 0,5 Гц, но с постоянным моментом на валу генератора. Колебания тока возбуждения эмитируют разницу в качестве выпрямленного тока. Результаты измерений представлены в таблице 1, зависимости коэффициентов несинусоидальности от частоты подаваемого тока на обмотку возбуждения представлены на рис. 3

Таблица 1

Результаты первой группы экспериментов

\begin{tabular}{|c|c|c|c|c|c|c|c|}
\hline \multirow{2}{*}{$f_{6030}, \Gamma_{ц}$} & \multirow{2}{*}{$f_{\text {cucm }}$, Гц } & \multicolumn{6}{|c|}{ THD } \\
\hline & & $U_{a}$ & $U_{b}$ & $U_{c}$ & $I_{a}$ & $I_{b}$ & $I_{c}$ \\
\hline 0,1 & 50,0228 & 0,499 & 0,584 & 0,484 & 4,622 & 3,106 & 2,633 \\
\hline 0,2 & 50,1085 & 0,476 & 0,482 & 0,369 & 1,660 & 1,521 & 1,398 \\
\hline 0,3 & 50,2288 & 0,310 & 0,134 & 0,309 & 0,182 & 0,563 & 0,473 \\
\hline 0,4 & 50,4551 & 0,410 & 0,260 & 0,315 & 0,269 & 0,778 & 0,773 \\
\hline 0,5 & 50,4503 & 0,415 & 0,191 & 0,410 & 0,210 & 0,741 & 0,702 \\
\hline
\end{tabular}

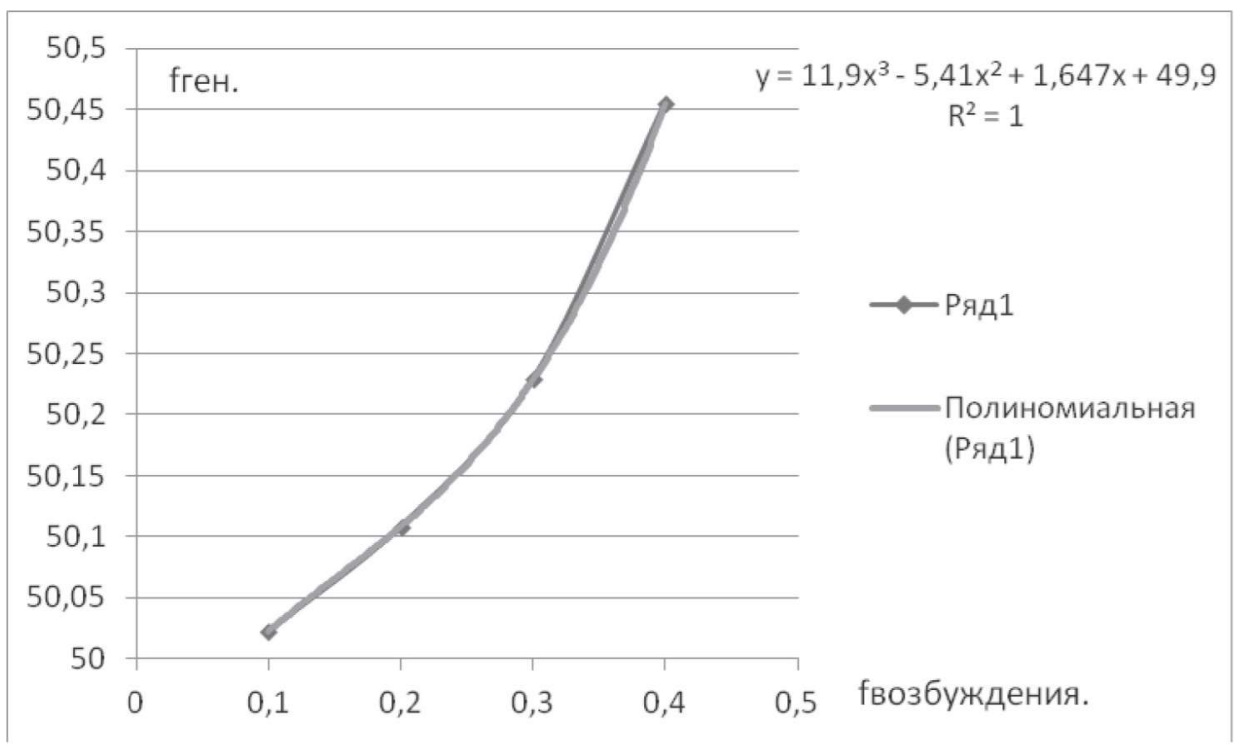

Рис. 3. Зависимость частоты генератора от частоты тока возбуждения при постоянстве приложенного момента

Очевидно влияние частоты тока возбуждения генератора на стабильность его работы. С увеличением коэффициента пульсации увеличивается частота генератора в виду появления реактивных составляющих в токе возбуждения $[4,5]$.

Результаты измерений представлены в таблице 2, зависимости коэффициентов несинусоидальности фазных напряжений и токов от частоты инжекции тока на обмотку возбуждения представлены на рис. 4 и 5. 


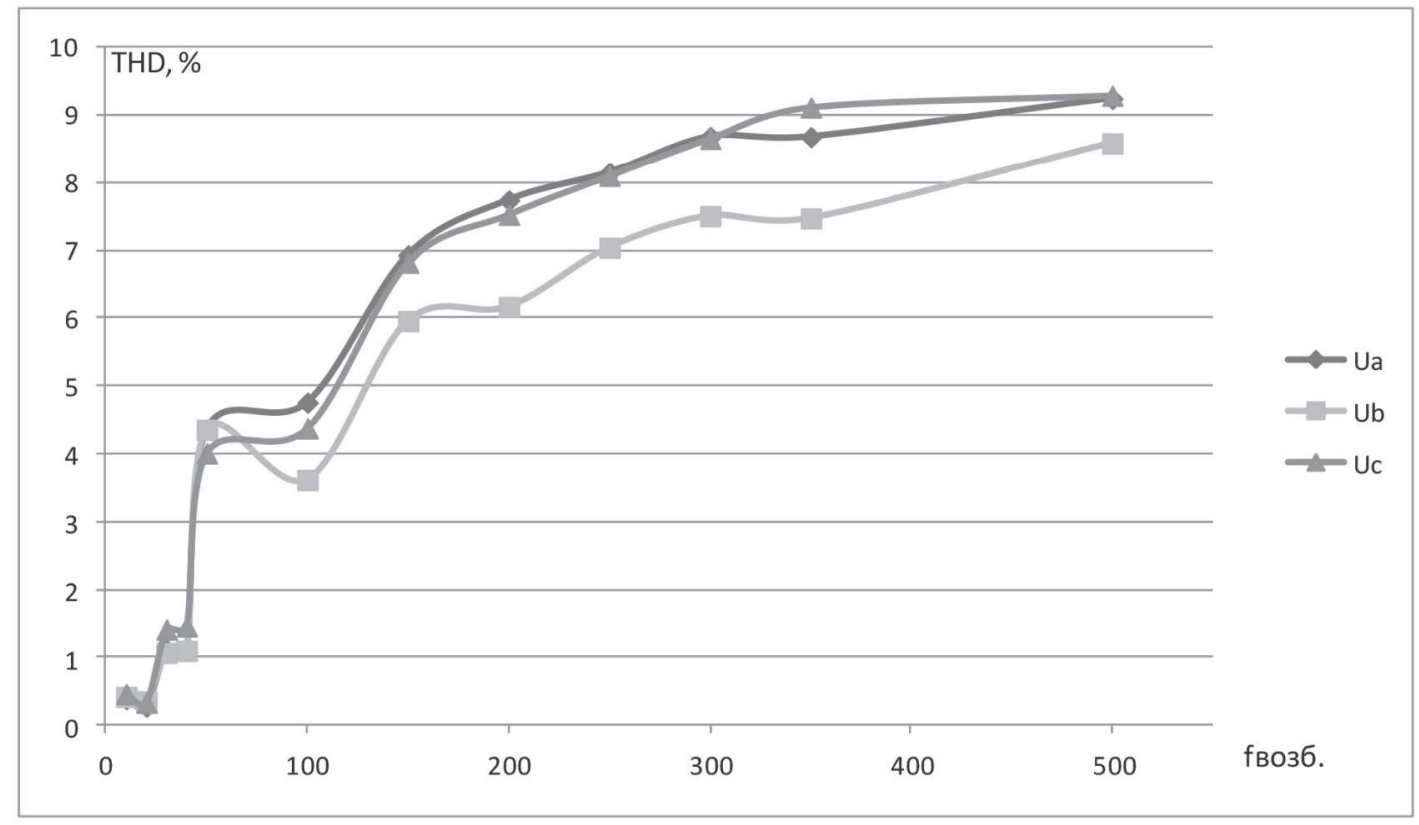

Рис. 4. Зависимости коэффициента не синусоидальности напряжения THD\% от частоты тока возбуждения для аварийного режима 2.

Второй эксперимент был проведен согласно схеме рис. 2a, где ток фазы b имитировал возникновение повреждения и инжекцию тока КЗ в диапазоне частот от 10 до 500 Гц при его постоянном действующем значении, и неизменности основного тока возбуждения формируемого по векторной сумме фазных токов А и С.

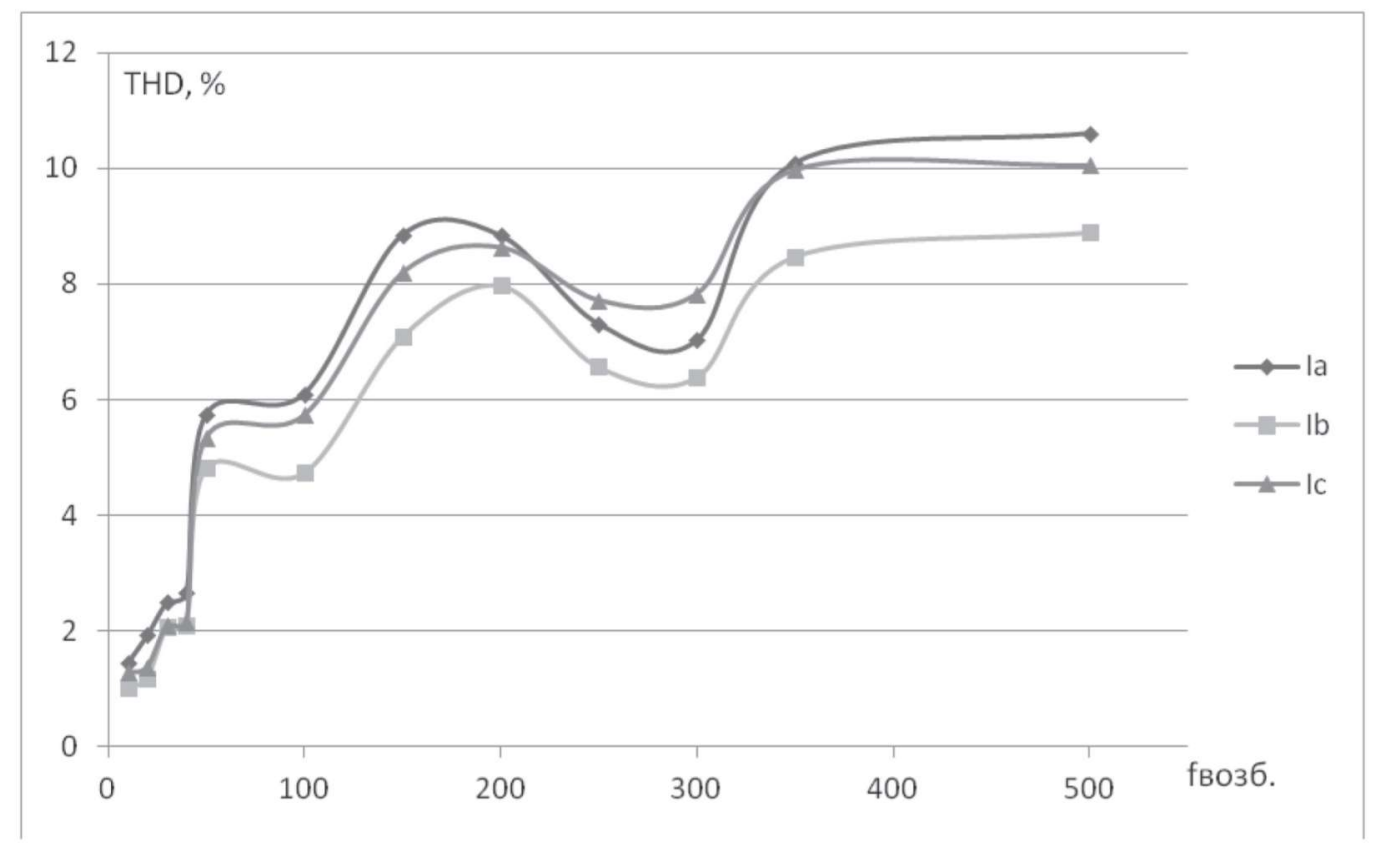

Рис. 5. Зависимости коэффициента не синусоидальности тока THD \% от частоты тока возбуждения для аварийного режима 2. 
Результаты второй группы экспериментов

\begin{tabular}{|c|c|c|c|c|c|c|c|c|}
\hline \multirow{2}{*}{$f_{60 s \bar{b}}, \Gamma \amalg$} & \multirow{2}{*}{$f_{\text {cucm }}, \Gamma_{\amalg}$} & \multicolumn{7}{|c|}{ THD, \% } \\
\hline & & $U_{a}, \mathrm{~B}$ & $U_{b}, \mathrm{~B}$ & $U_{c}, \mathrm{~B}$ & $I_{a}, \mathbf{A}$ & $I_{b}, \mathrm{~A}$ & $I_{c}, \mathrm{~A}$ & $I_{6050}, \mathrm{~A}$ \\
\hline 10 & 49,9062 & 0,390 & 0,423 & 0,464 & 1,452 & 1,033 & 1,285 & 7,832 \\
\hline 20 & 49,8011 & 0,277 & 0,357 & 0,336 & 1,934 & 1,191 & 1,386 & 6,184 \\
\hline 30 & 49,9809 & 1,115 & 1,069 & 1,413 & 2,499 & 2,069 & 2,102 & 4,644 \\
\hline 40 & 49,9871 & 1,140 & 1,100 & 1,460 & 2,665 & 2,113 & 2,168 & 4,215 \\
\hline 50 & 49,984 & 4,357 & 4,357 & 4,009 & 5,749 & 4,819 & 5,346 & 11,084 \\
\hline 100 & 49,9893 & 4,756 & 3,617 & 4,377 & 6,105 & 4,738 & 5,736 & 5,301 \\
\hline 150 & 49,9764 & 6,926 & 5,958 & 6,818 & 8,842 & 7,092 & 8,190 & 3,554 \\
\hline 200 & 49,9793 & 7,736 & 6,165 & 7,524 & 8,837 & 7,978 & 8,638 & 3,225 \\
\hline 250 & 49,9625 & 8,146 & 7,038 & 8,098 & 7,311 & 6,569 & 7,723 & 0,192 \\
\hline 300 & 49,9228 & 8,679 & 7,505 & 8,652 & 7,027 & 6,391 & 7.832 & 1,920 \\
\hline 350 & 49,956 & 8,676 & 7,470 & 9,114 & 10,088 & 8,480 & 9,973 & 0,451 \\
\hline 500 & 50,0017 & 9,243 & 8,586 & 9,288 & 10,599 & 8,899 & 10,051 & 0,412 \\
\hline
\end{tabular}

При анализе зависимостей, изображенных на рис. 2, видно, что коэффициенты несинусоидальности для токов и напряжений стремительно увеличиваются с ростом частоты подаваемого на обмотку возбуждения тока. Данная тенденция сохраняется до достижения частоты 350 Гц, при дальнейшем повышении частоты скорость нарастания THD \% значительно снижается.

Заключение / Conclusion. По результатам проведенных экспериментов можно сделать вывод о зависимости параметров синхронного генератора от работы системы возбуждения не только по показателям быстродействия и кратности форсировки, но и по качеству подаваемого сигнала тока возбуждения. Как показала первая серия опытов, присутствие в токе возбуждения низкочастотных составляющих, очевидно, окажет влияние на процесс регулирования частоты и реактивной мощности, обеспечиваемых генератором. Такое присутствие создает излишнюю реакцию систем регулирования напряжения и реактивной мощности. Наглядно излишнее изменение частоты иллюстрируют зависимости частоты генератора от пульсаций тока возбуждения (рис. 3). Возникновение же ситуаций высокочастотных составляющих в контуре тока возбуждения порождает общий тренд ухудшения коэффициентов несинусоидальности токов и напряжений, о чем свидетельствуют данные, полученные в результате проведенных экспериментов. Таким образом, контроль показателей качества электроэнергии на шинах генераторного напряжения не только косвенно повлияет на снижение потерь в электрических сетях [6], но и позволит диагностировать повреждения в обмотках возбуждения синхронных генераторов и системах их питания и управления.

\section{ЛИТЕРАТУРА И ИНТЕРНЕТ-РЕСУРСЫ}

1. ГОСТ 32144-2013 Электрическая энергия. Совместимость технических средств электромагнитная. Нормы качества электрической энергии в системах электроснабжения общего назначения.

2. Gigavat URL: http://www.gigavat.com/generator vozbughdenie.php (дата обращения: 01.11.2018).

3. SolverBook URL: http://ru.solverbook.com/spravochnik/koefficienty/ (дата обращения: 01.11.2018).

4. Generator Excitation Systems Sensitivity Analysis and Their Model Parameter's Reduction / Iman Naziri Moghaddam, Zia Salami, Saeed Mohajeryami // Energy Production and Infrastructure (EPIC) Electrical and Computer Engineering, University of North Carolina at Charlotte Charlotte, NC 28223, USA. Email: \{inazirim, zsalami, smohajer \}@uncc.edu. 
5. Analytical Analysis of the Variable Frequency Power System with Shunt-Connected Voltage Regulator / D. Makarov, G. Zinoviev, S. Kharitonov // Dept. of Electronics and Electrical Engineering. Novosibirsk State Technical University. Novosibirsk, Russian Federation.

6. Петров А. В., Костюков Д. А., Звада П. А. Экспериментальное исследование скин-эффекта в самонесущих изолированных проводах // Вестник СКФУ. 2018. № 3 (66). 209 с.

7. Каталог OMICRON. Описание и назначение. URL: http://ekra.nt-rt.ru/images/showcase/CMLineCatalog-RUS.pdf (дата обращения: 10.03.2018).

8. Инструкция по эксплуатации NI 9225. URL: http://russia.ni.com/sites/default/files/NI-9225-rus.pdf. (дата обращения: 01.11.2018).

9. Преобразователи напряжения измерительные аналого-цифровые модульные NI 9229, NI 9239. URL: http://russia.ni.com/sites/default/files/\%D0\%A0\%D0\%AD\%20NI\%209229-9239.pdf. (дата обращения: 01.11.2018).

10. Oppenheim A.V., Schafer R.W. Discrete-Time Signal Processing. 3rd edition. - Prentice Hall, 2009. - 1120 p. (Prentice Hall Signal Processing).

11. ГОСТ IEC 61000-4-30-2017 Электромагнитная совместимость (ЭМС) Часть 4-30 МЕТОДЫНСПЫТАНИЙ И ИЗМЕРЕНИЙ Методы измерений качества электрической энергии. М.: Стандартинформ, 2018 г.

\section{REFERENCES AND INTERNET RESOURCES}

1. GOST 32144-2013 Jelektricheskaja jenergija. Sovmestimost' tehnicheskih sredstv jelektromagnitnaja (Electrical energy. Electromagnetic compatibility of technical equipment) Normy kachestva jelektricheskoj jenergii v sistemah jelektrosnabzhenija obshhego naznachenija.

2. Gigavat URL: http://www.gigavat.com/generator_vozbughdenie.php (data obrashhenija: 01.11.2018.

3. SolverBook URL: http://ru.solverbook.com/spravochnik/koefficienty (data obrashhenija: 01.11.2018.

4. Generator Excitation Systems Sensitivity Analysis and Their Model Parameter's Reduction / Iman Naziri Moghaddam, Zia Salami, Saeed Mohajeryami // Energy Production and Infrastructure (EPIC) Electrical and Computer Engineering, University of North Carolina at Charlotte Charlotte, NC 28223, USA Email: \{inazirim, zsalami, smohajer \} @uncc.edu.

5. Analytical Analysis of the Variable Frequency Power System with Shunt-Connected Voltage Regulator / D. Makarov, G. Zinoviev, S. Kharitonov // Dept. of Electronics and Electrical Engineering. Novosibirsk State Technical University. Novosibirsk, Russian Federation.

6. Petrov A. V., Kostjukov D. A., Zvada P. A. Jeksperimental'noe issledovanie skin-jeffekta v samonesushhih izolirovannyh provodah (Experimental study of the skin effect in self-supporting installations). Vestnik SKFU, nauchnyj zhurnal, gl. red. V. N. Parahina., 2018, No3 (66), 7-14 pp.

7. Katalog OMICRON. Opisanie i naznachenie. URL: http://ekra.nt-rt.ru/images/showcase/CM-LineCatalogRUS.pdf (data obrashhenija: 10.03.2018).

8. Instrukcija po jekspluatacii NI 9225. URL: http://russia.ni.com/sites/default/files/NI-9225-rus.pdf. (data obrashhenija: 01.11.2018).

9. Preobrazovateli naprjazhenija izmeritel'nye analogo-cifrovye modul'nye NI 9229, NI 9239. URL: http:// russia.ni.com/sites/default/files/\%D0\%A0\%D0\%AD\%20NI\%209229-9239.pdf. (data obrashhenija: 01.11.2018).

10. Oppenheim A. V., Schafer R. W. Discrete-Time Signal Processing. 3rd edition. Prentice Hall, 2009. 1120 p. (Prentice Hall Signal Processing).

11. GOST IEC 61000-4-30-2017 Jelektromagnitnaja sovmestimost' (JeMS) Chast' 4-30 METODYISPYTANIJ I IZMERENIJ Metody izmerenij kachestva jelektricheskoj jenergii. M.: Standartinform, 2018.

\section{СВЕДЕНИЯ ОБ АВТОРАХ}

Звада Павел Александрович, старший преподаватель кафедры автоматизированных электроэнергетических систем и электроснабжения инженерного института СКФУ. E-mail: zpass1781@mail.ru

Пляико Максим Сергеевич, студент 1 курса магистратуры кафедры автоматизированных электроэнергетических систем и электроснабжения инженерного института СКФУ. E-mail: BEL-8008@yandex.ru

Миронов Андрей Валерьевич, студент 1 курса магистратуры кафедры автоматизированных электроэнергетических систем и электроснабжения инженерного института СКФУ. E-mail: like10@mail.ru 


\section{INFORMATION ABOUT AUTHORS}

Pavel Zvada, Senior lecturer of the Chair of Automatic Electrical Power Systems and Electric Power Supply, Engineering Institute, NCFU. E-mail: zpass1781@mail.ru

Maxim Plyashko, 1st year student of the magistracy of the department of automated electric power systems and power supply of the engineering institute of NCFU. E-mail: BEL-8008@yandex.ru

Andrey Mironov, 1st year student of the magistracy of the department of automated electric power systems and power supply of the engineering institute of NCFU. E-mail: like10@mail.ru

\section{ОЦЕНКА ВКЛАДА ПОТРЕБИТЕЛЯ В НЕСИММЕТРИЮ НАПРЯЖЕНИЙ ПО НУЛЕВОЙ ПОСЛЕДОВАТЕЛЬНОСТИ В ТОЧКЕ ОБЩЕГО ПРИСОЕДИНЕНИЯ}

В статье представлена методика оценки вклада потребителя в уровень несимметрии напряжений по нулевой последовательности в точке обчего присоединения. Приведены результатьы вычислительного эксперимента, определяючие возможность использования в качестве критерия оценки уровень активной составляющей моцности нулевой последовательности. Показано, что оценка долевого вклада потребителя в несимметрию напряжения по нулевой последовательности может быть осуиествлена посредством векторных измерений фазных напряжсении и тока нулевого провода.

Ключевые слова: несимметрия, сеть с глххозаземленюой нейтралью, нулевая последовательность, смецение нейтрали, долевой вклад потребителя, моиность искажения, вычислительный эксперимент.

\section{Dmitrii Kostyukov \\ ASSESSMENT OF CONSUMER IMPACT ON VOLTAGE ASYMMETRY \\ BY THE ZERO SEQUENCE AT THE POINT OF COMMON CONNECTION}

The article presents a technique for assessment the level of consumers' impact on the voltage asymmetry by the zero sequence at the point of common connection. The results of a computational experiment that determine the possibility of using the level of the active component of the zero sequence distortion power as a criteria of assessment are presented. It is shown that the evaluation of the consumers' impact on the voltage asymmetry by the zero sequence can be carried out by means of vector measurements of phase voltages and current of the neutral wire.

Key words: asymmetry, solidly grounded neutral system, zero sequence, neutral displacement, share contribution of consumer, distortion power, computational experiment.

Bведение / Introduction. Режимы работы современных низковольтных сетей характеризуются относительно низким уровнем качества электрической энергии (КЭЭ). Это в первую очередь связано с тем, что в большинстве случаев ухудшение отдельных показателей качества электроэнергии (ПКЭ) обусловлено воздействием потребителя на питающую сеть. Данное утверждение было зафиксировано в утратившем силу стандарте [1], определяющем требования к КЭЭ, однако в действующем стандарте [2] вопросы выявления виновника ухудшения КЭЭ не затрагиваются. При этом следует отметить, что проблема КЭЭ в последние годы не только не потеряла актуальность, но и имеет некоторую тенденцию к обострению [3-6]. 